\title{
Co-infection with stealth adapted viruses may explain zika virus associated microcephaly
}

\begin{abstract}
The premise of this paper is that the apparent teratogenicity of Zika virus may result from co-infection of the mothers with stealth adapted viruses. Specific testing should, therefore, be performed on mothers and their affected infants for stealth adapted viruses. Even if infection is not confirmed, extended studies on stealth adapted viruses can provide important insights into many additional neuropsychiatric illnesses, including autism. These endeavors can also help expedite the development and evaluation of anti-virus therapies based upon enhancing the alternative cellular energy (ACE) pathway. Particular emphasis can be given to the potential clinical benefit of consuming water activated with KELEA (kinetic energy limiting electrostatic attraction). Indeed, urgent consideration should be given to having all pregnant women at risk for Zika virus infection to begin consuming KELEA activated water.
\end{abstract}

Keywords: Zika, ACE, Microcephaly, KELEA, Vaccine, Stealth adapted virus, Water, Autism, Cytomegalovirus, Enerceutical ${ }^{\mathrm{TM}}$, moringa oleifera
Volume 3 Issue I - 2016

\author{
W John Martin \\ Institute of Progressive Medicine, USA \\ Correspondence: W John Martin, Institute of Progressive \\ Medicine, 1634 Spruce Street, South Pasadena CA 91030, USA, \\ Tel 626-616-2868, Email wjohnmartin@ccid.org
}

Received: February II, 2016 | Published: February II, 2016
Abbreviations: ACE, Alternative Cellular Energy; KELEA, Kinetic Energy Limiting Electrostatic Attraction; HSV, Herpes Simplex Virus; HZV, Herpes Zoster Virus; HPV, Human Papillomavirus Virus; HIV, Human Immunodeficiency Virus

\section{Introduction}

The apparent association of Zika virus infection with microcephaly appears to be largely confined to Brazil. While this could be due to genetic changes in the Brazilian Zika virus, it is also consistent with a facilitating effect of co-infection with another virus. Public health authorities have been reluctant to acknowledge the presence of stealth adapted viruses. ${ }^{1}$ These viruses have deletions or mutations in the relatively few components in viruses that are normally targeted by the cellular immune system. ${ }^{2}$ Consequently, stealth adapted viruses do not evoke an inflammatory response; the hallmark of an infectious process. $^{3}$

The political reluctance to pursue stealth adapted viruses arose because some of the viruses were unequivocally derived from cytomegaloviruses of monkeys used to produce polio vaccines. ${ }^{4}$ Moreover, cytomegalovirus contaminated polio vaccines tested in Africa may well explain the origin of HIV. ${ }^{5}$ Cytomegaloviruses can infect the placenta and can promote the replication of other viruses in normally non-permissive cells. ${ }^{6}$ Conversely, other viruses can enhance the replication of cytomegaloviruses. ${ }^{7}$ It would be useful if public health authorities studying the Zika virus, extended their efforts to detecting stealth adapted viruses. This can most readily be accomplished using virus culture methods. ${ }^{1}$ Animals and possibly insects are also susceptible to certain stealth adapted viruses. ${ }^{4,8}$

\section{Discussion}

Additional benefits will arise from such studies. First, more widespread testing will confirm that stealth adapted viruses can cause autism in children ${ }^{9}$ as well as various neuropsychiatric illnesses in adults. ${ }^{3,10}$ Second, directed studies will show the potential role of routine vaccinations in provoking stealth adapted virus infections. This is relevant in view of the Brazilian government earlier decision to administer tetanus, diphtheria, and acellular pertussis vaccine (Tdap) to all pregnant women. ${ }^{11}$ It is reasonable to exclude those infected with stealth adapted viruses from routine vaccination programs, especially during pregnancy..$^{12}$ Third, and most importantly, it will highlight the alternative cellular energy (ACE) pathway as an effective nonimmunological defense mechanism against both stealth adapted and conventional viruses. Indeed, preliminary studies have confirmed the clinical efficacy of enhancing the ACE pathway in the suppression of herpes simplex virus (HSV), herpes zoster virus (HZV), human papillomavirus (HPV) and human immunodeficiency virus (HIV) infections. ${ }^{13-15}$ ACE pathway therapy can also be used to suppress stealth adapted virus infections. ${ }^{16}$

The ACE pathway is mediated by a dynamic (kinetic) energy of the body's fluids. ${ }^{17}$ It results from the absorption of a natural environmental force termed KELEA (kinetic energy limiting electrostatic attraction). The fundamental role of this force is probably to prevent the fusion and annihilation of electrostatically attracted opposite electrical charges. KELEA leads to a loosening of intermolecular hydrogen bonding of fluid molecules, ${ }^{18}$ as reflected in lower surface tension and in increased volatility. KELEA can also directly contribute kinetic activity and other forms of energy to fluid molecules.

Water can be activated by the addition of KELEA attracting compounds, including many simple dipolar compounds and enerceutical ${ }^{\mathrm{TM}}$ foods, including moringa oleifera. ${ }^{19}$ The activating compounds can subsequently be removed by either zero-residue filtration or repeated dilutions, as in homeopathy. Water can also be activated by being placed in the vicinity of KELEA attracting energy fields. ${ }^{20-22}$ Consuming KELEA activated water would appear to provide a convenient means of enhancing the ACE pathway. Urgent efforts should, therefore, be undertaken to evaluate this option in the face of emerging and existing virus infections.

\section{Conclusion}

The emergence of new virus infections poses a recurring threat to mankind. Worldwide attention is currently focused on the occurrence of microcephaly in newborn infants in Brazil. The transplacental passage of Zika virus and subsequent brain damage may involve co-infection with stealth adapted viruses. Moreover, the severity of 
the stealth adapted virus infection may have been provoked in early pregnancy by the administration of Tdap vaccine. The opportunity exists for public health officials to evaluate published data relating to stealth adapted viruses and to a non-immunological, anti-virus defense mechanism mediated through the alternative cellular energy (ACE) pathway. Even while waiting for the results of controlled clinical, animal and laboratory studies, urgent consideration should be given to providing KELEA activated water to pregnant women in Zika endemic areas.

\section{Acknowledgements}

The Institute of Progressive Medicine is a component of MI Hope Inc., a non-profit public charity.

\section{Conflicts of interest}

None.

\section{References}

1. Martin WJ, Zeng LC,Ahmed K, et al. Cytomegalovirus-related sequences in an atypical cytopathic virus repeatedly isolated from a patient with the chronic fatigue syndrome. Am J Pathol. 1994;145(2):440-451.

2. Martin WJ. Stealth adaptation of viruses: Review and updated molecular analysis on a stealth adapted African green monkey simian cytomegalovirus (SCMV). Journal of Human Virology \& Retrovirology. 2014;1(4):00020.

3. Martin WJ. Severe stealth virus encephalopathy following chronic fatigue syndrome-like illness: Clinical and histopathological features. Pathobiology. 1996;64(1):1-8.

4. Martin WJ, Ahmed KN, Zeng LC, et al. African green monkey origin of the atypical cytopathic 'stealth virus' isolated from a patient with chronic fatigue syndrome. Clin Diag Virol. 1995;4(1):93-103.

5. Martin WJ. Chimpanzees inoculated with cytomegalovirus contaminated polio vaccines may explain origin of HIV-1. Journal of Human Virology \& Retrovirology. 2015;2:00035.

6. Winklhofer KF, Albrecht I, Wegner M, et al. Human cytomegalovirus immediate-early gene 2 expression leads to JCV replication in nonpermissive cells via transcriptional activation of JCV T antigen. Virology. 2000;275(2):323-334.

7. Krommelbein N, Wiebusch L, Schiedner G, et al. Adenovirus E1A/ E1B transformed amniotic fluid cells support human cytomegalovirus replication. Viruses. 2016;8(2):E37.

8. Martin WJ, Glass RT. Acute encephalopathy induced in cats with a stealth virus isolated from a patient with chronic fatigue syndrome. Pathobiology. 1995;63(3):115-118.
9. Martin WJ. Stealth virus isolated from an autistic child. J Aut Dev Dis. 1995;25(2):223-224.

10. Martin WJ. Stealth adapted viruses. A bridge between molecular virology and clinical psychiatry. Open J Psychiatry. 2015;5(4):311-319.

11. Brasil Ministério da: A página solicitada não pôde ser encontrada. Portal da Saúde. 2014.

12. Martin WJ. Stealth Adaptation of Viruses: Implications for Therapy and for Potential Toxicity of Vaccines. J Virol Curr Res. 2015;1(1):555551.

13. Martin WJ, Stoneburner J. Symptomatic relief of herpetic skin lesions utilizing an energy based approach to healing. Exp Mol Pathol. 2005;78(2):131-134.

14. Martin WJ, Stoneburner J. Alternative cellular energy (ACE) pathway Activation as the mode of action of neutral red dye phototherapy of human viruses. J Hum Virol Retrovirol. 2014;1(4):00019.

15. Dubrov V, Dubrova T, Christner D, et al. Alternative Cellular Energy Based Therapy using EnercelÔ in Advanced AIDS Patients Co-infected with Tuberculosis and Treated in Chernigov, Ukraine. J Hum Virol Retrovirol. 2015;2(6):00061.

16. Martin WJ. Alternative cellular energy (ACE): activation as natural therapy for autism. In Stealth Adapted Viruses; Alternative Cellular Energy (ACE) \& KELEA Activated Water. Author House, USA. 2014;pp.87-102.

17. Martin WJ. Alternative cellular energy pathway therapy using KELEA activated water. International J Complementary \& Alternative Medicine. 2015;2(2):00051.

18. Martin WJ. KELEA: A natural energy that seemingly reduces intermolecular hydrogen bonding in water and other liquids. Open Journal of Biophysics. 2015;5(3):69-79.

19. Martin WJ. Do the benefits of Moringa oleifera trees extend to KELEA activation of water? Advances in Plants \& Agriculture Research. 2015;2(1):00036.

20. Martin WJ. Interacting light paths attract KELEA (kinetic energy limiting electrostatic attraction) and can lead to the activation of water. Open Journal of Biophysics. 2015;5(4):115-121.

21. Martin WJ. Interactive electric fields can attract KELEA (kinetic energy limiting electrostatic attraction) and can lead to the activation of water. International $J$ Complementary \& Alternative Medicine. 2015;1(6):00034.

22. Martin WJ. KELEA Activation of Water and Other Fluids for Health, Agriculture and Industry. Journal of Water Resource and Protection. 2015;7(16):1331-1344. 